\title{
Perceived Risk and Mental Health Problems among Healthcare Professionals during COVID-19 Pandemic: Exploring the Mediating Effects of Resilience and Coronavirus Fear
}

\author{
Murat Yıldırım ${ }^{1,2}$ (D) Gökmen Arslan ${ }^{3} \cdot$ Ahmet Özaslan ${ }^{4}$ \\ Accepted: 24 October 2020 / Published online: 16 November 2020 \\ (C) Springer Science+Business Media, LLC, part of Springer Nature 2020
}

\begin{abstract}
During coronavirus (COVID-19) pandemic, healthcare professionals were particularly at high-risk of developing symptoms of mental health problems due to being on the frontline in the battle against COVID-19. This study examined the mediating roles of resilience and coronavirus fear in the relationship between perceived risk and mental health problems among healthcare professionals including doctors and nurses who were actively treating patients confirmed with COVID-19. We recruited 204 healthcare professionals (50\% females) with a mean age of 32.92 years $(S D=7.01)$. Results showed that perceived risk and coronavirus fear positively predicted depression, anxiety, and stress while resilience negatively predicted those mental health problems. Coronavirus fear mediated the relationship between perceived risk and resilience, depression, anxiety, and stress. Additionally, resilience mitigated the effect of coronavirus fear on depression, anxiety, and stress. This study is among the first indicating the importance of resilience and fear as a critical mechanism that explains the relationship between perceived risk and mental health problems among health professionals directly caring for COVID-19 patients.
\end{abstract}

Keywords COVID-19 Mental health problems · COVID-19 perceived risk · Resilience COVID-19 fear $\cdot$ Healthcare professionals

\section{Murat Yildirım}

muratyildirim@agri.edu.tr; muratyildirimphd@gmail.com

1 Department of Psychology, Faculty of Science and Letters, Ağrı İbrahim Çeçen University, Erzurum Yolu 4 Km, 04100 Ağrı, Turkey

2 Department of Neuroscience, Psychology and Behaviour, University of Leicester, Leicester, UK

3 Department of Psychological Counseling and Guidance, Faculty of Education, Mehmet Akif Ersoy University, Burdur, Turkey

4 Department of Child Mental Health and Diseases, Gazi University, Ankara, Turkey 
Globally, as of 10 October 2020, more than 36.8 million people have been affected by COVID-19 with $1.067,114$ deaths, while Turkey registered 332.382 confirmed cases of COVID-19 and 8.722 deaths (Center for Systems Science and Engineering 2020). As in many other countries, the Turkish government implemented a wide range of COVID-19-related measures to reduce the spread of the COVID-19 such as partial lockdown, travel restrictions, staying home, and withdrawal from close social contact with friends and relatives outside their household except for essentials (Yıldırım and Arslan 2020). With the emergence of COVID19 , people can develop various mental health problems or face with worsening existing health conditions. Although the experience of mental health problems is an inevitable part of life, it may become more severe and prevalent among health care professionals during the pandemic as they are on the frontline fighting COVID-19. This can impact their life to a great extent that in-depth research is required to promote their mental health. Despite the findings on the relationship between perceived risk and mental health problems during pandemic (Bults et al. 2011), there is a dearth of studies on the mechanism underlying this relationship. This study primarily focused on the mediation effects of resilience and fear in the relationship between perceived risk and mental health problems (Fig. 1).

Healthcare professionals facing unexpected and unknown events typically experience various stress reactions. Due to working closely to help those infected with COVID-19, healthcare professionals are at a greater risk than any others to develop symptoms of psychological problems including anxiety, panic, or other stress-related disorders (Chen et al. 2020). Such symptoms can be derived from various sources such as excessive workloads, lack of personal protective equipment, exposure to negative news content about COVID-19, and inadequate support from mental health professionals (Cai et al. 2020). Although limited, recent reports have demonstrated that the prevalence of mental health problems among healthcare workers during COVID-19 was estimated as $14.5 \%$ for anxiety, $8.9 \%$ for depression, $6.6 \%$ for stress, and $7.7 \%$ for clinical concern of PTSD in Singapore (Tan et al. 2020). High levels of anxiety,

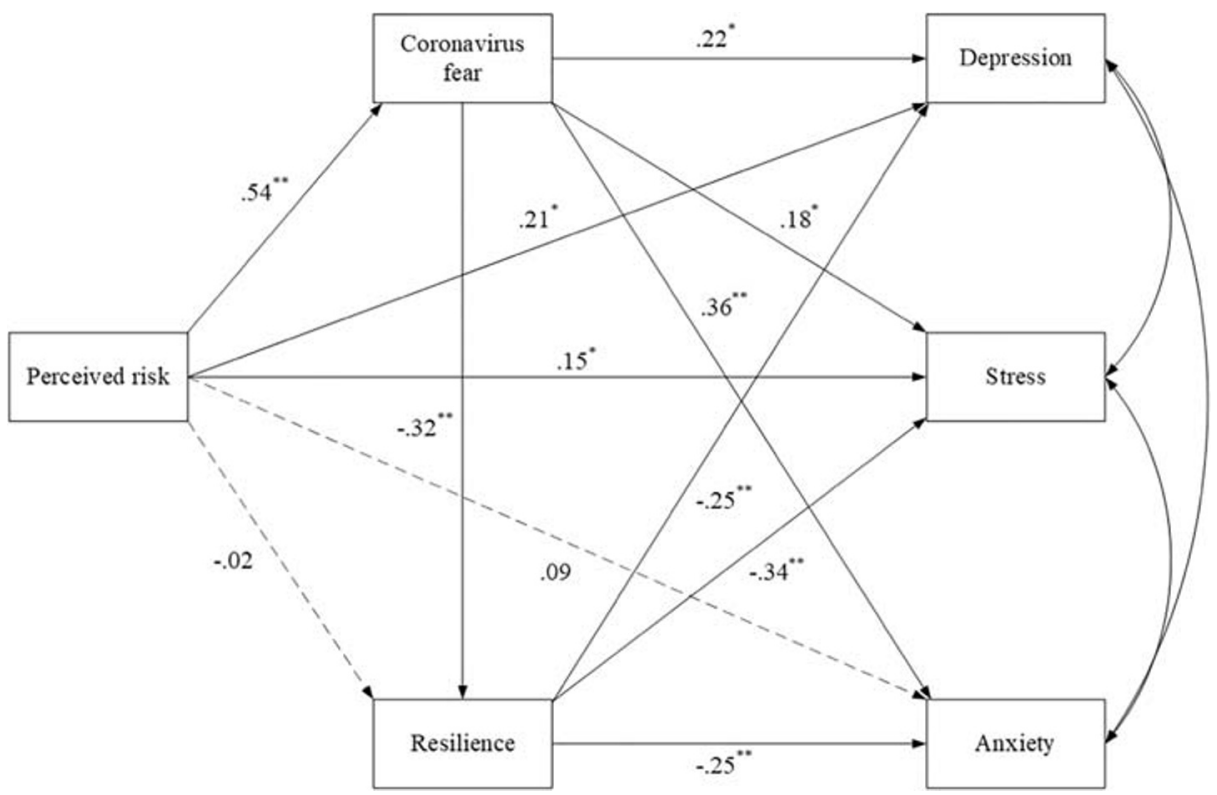

Fig. 1 Structural model demonstrating the association between the variables of the study 
stress, and depression among medical staff and medical students have been also reported in Iran (Ashtari et al. 2020) and China (Huang and Zhao 2020) during the pandemic. Furthermore, evidence on other infectious diseases such as SARS, MERS, and Ebola showed that many healthcare professionals experience mental health problems during the outbreak (Lancee et al. 2008; Peeri et al. 2020). For example, healthcare professionals suffer from high prevalence of psychiatric disorders (30\%), including burnout, anxiety, depressive symptoms, and traumatic stress, even after the SARS outbreak (Lancee et al. 2008). During the SARS and MERS outbreaks, the incident rate of healthcare professionals was respectively $21 \%$ and $18.6 \%$, which caused severe emotional problems such as anxiety and depression (Peeri et al. 2020). However, there have been several studies that have examined the roles of psychological resources that people can use to mitigate the adverse effects of a pandemic on psychological health. Resilience, dispositional hope, meaningful living, and positive emotions have been shown to manifest positive effects on psychological health problems (Arslan et al. 2020).

Perceived risk plays a key role in shaping individuals' health-related behaviours (Janz and Becker 1984). Higher perceived risk is associated with higher experience of fear (Jackson 2011; Warr 2000). The risk-resilience model (Masten 2001) has emphasized that perceived risk in the face of adversity heightens the tendency of negative outcomes and resilient individuals can turn negative outcomes into positive outcomes. During COVID-19, perceived risk and severity of the virus were associated with poor mental health outcomes ( $\mathrm{Li}$ et al. 2020) and greater tendency to practice preventive behaviours against COVID-19 (Khosravi 2020; Wise et al. 2020). Another study reported high levels of perceived risk, perceived susceptibility, perceived severity, worry, general anxiety, and disruption in daily life due to COVID-19 (Kwok et al. 2020). Similar results have been reported from other infectious diseases, including SARS and Ebola; higher perceived risk was associated with greater mental health problems (Cheng et al. 2006; Yang and Chu 2018).

Fear of COVID-19 has become a global health threat. Fear is conceptualised as an unpleasant emotion accompanied by excessive levels of emotive avoidance concerning particular stimuli (Perin et al. 2015). While the experience of fear to some extent can be helpful for people in terms of leading them to engage in protective behaviours against COVID-19, extreme levels of fear can be detrimental to psychological and physical health (Garfin et al. 2020; Sloan et al. 2020) both at individual and societal levels (Mertens et al. 2020).

Concerning COVID-19, although limited, prior work demonstrates significant impact of fear on psychological and behaviours change, with higher levels of mental health problems (Ahorsu et al. 2020) and engagement in preventive behaviours such as social distancing and improved handwashing (Harper et al. 2020). Sloan et al. (2020) demonstrated that people who have high levels of personal fear of COVID-19 reported greater mental health harm while fear for others was not related with decreased mental health. In times of crisis, extreme fear and uncertainty might turn into various mental health concerns, including distress reactions (excessive fear of illness, insomnia, anger), health risk behaviours (social withdrawal, excessive use of alcohol and tobacco), mental health disorders (anxiety disorders, depression, posttraumatic stress disorder, somatization), and poor perceived health (Shigemura et al. 2020).

Resilience is an essential resource used by many people in times of adversity. Resilience can be described as the capability of "bounce back" from hardship quickly and adapt to new situation flexibly and even psychological growth in the face of significant adversity (Bonanno 2004; Smith et al. 2008). Earlier research on healthcare professional resilience has primarily focused on avoidance of burnout which is typically related to workplace stress (Fertleman and Carroll 2013). During coronavirus crisis, healthcare professionals may face with various 
challenges that can affect their resilience and mental health. According to Robertson et al. (2016), healthcare professionals in primary care can be overwhelmed by different sources of challenges such as difficult clinical problems, conflict with challenging patients, and organisational issues. Resilient healthcare professionals can preserve a positive outlook towards the patients, fulfil their duties realistically, and have effective strategies to reduce stress despite these challenges (Stevenson et al. 2011). In wider literature, resilience has been found to be a strong predictor of psychological distress and quality of life (Tecson et al. 2019) and subjective, psychological well-being, and mental health (Ylldırım 2019). Previous research has also identified mediator role of resilience between various psychological factors and subjective well-being and psychological health in the context of COVID-19 (Ylldırım and Arslan 2020), suggesting that resilience can be a great source of mental health as it buffers the detrimental effects of stress on psychological health (Ong et al. 2006). Thus, it is plausible to assume that resilience can mediate the relationship between risk factors (e.g. fear, perceived risk) and mental health problems among healthcare professionals.

There is a paucity of evidence on the potential mediation of resilience and fear in the relationship between perceived risk and mental health, particularly among health care professionals during the pandemic. As such, this study examined the potential mediating effects of resilience and fear in the relationship between perceived risk and mental health problems of health professionals who were actively treating patients confirmed with COVID-19. Understanding the underlying mechanism between perceived risk and mental health problems can inform the tailoring and implementation of effective intervention programmes targeted at increasing resilience and reducing the mental health problems of health care professionals caring for COVID-19 patients. We hypothesised that (a) perceived risk would have a direct effect on resilience, coronavirus fear, and mental health problems; (b) resilience and fear would have direct effects on mental health problems; (c) coronavirus fear would mediate the relationship between perceived risk and resilience; (d) resilience would mediate the relationship between fear and mental health problems; and (e) fear would mediate the relationship between perceived risk and mental health problems.

\section{Method}

\section{Participants}

The sample of this study was formed with 204 (50\% female; $\left.\mathrm{M}_{\mathrm{age}}=32.92 \pm 7.01\right)$ healthcare professionals who took an active role in the departments of COVID-19 inpatient clinics, outpatient clinics, or intensive care units in Turkey. In total, $47.55 \%$ of participants were doctors, $22.06 \%$ were nurses, and $30.39 \%$ were other care workers (e.g. medical assistant). Other prominent demographic characteristics were $67.16 \%$ married, $55.39 \%$ university graduate, $76 \%$ without a chronic disease, and $31.37 \%$ with three households.

\section{Measures}

Mental Health Depression Anxiety Stress Scales-21 (DASS-21) was used to assess negative emotional states (Lovibond and Lovibond 1995). The DASS-21 includes 21 items containing three subscales ( 7 items per subscale): Depression, Anxiety, and Stress. The scale is based on a 4-point Likert-type, where 0 represents "did not apply to me at all" to 3 representing "applied 
to me very much or most of the time". Higher scores are associated with high depression, anxiety, and stress. Good evidence of reliability and validity has been reported in Turkish (Sariçam 2018).

Resilience The Brief Resilience Scale (BRS) was used to measure one's capability to bounce back from or overcome stressful situations (Smith et al. 2008). The BRS includes 6 items rated on a 5-point Likert type, ranging from 1 (strongly disagree) and 5 (strongly agree). Higher scores indicate high resilience. Sound psychometric properties for the BRS were reported in the Turkish language (Doğan 2015).

Risk Perceived risk was measured with two questions: "Compared to most people your age and sex, what would you say your chances are for developing coronavirus?" (Weinstein 1982) and "How risky would it be for you if you think that you have coronavirus symptoms?". Participants were asked to rate each question using a 5-point Likert response format, ranging from 1 (much lower than average) to 5 (much higher than average). Higher scores signify greater perceived risk.

Fear Coronavirus fear was assessed with three items: "I am fear of coronavirus", "I am frightened by coronavirus", and "I am very concerned about catching coronavirus." Each item is rated on a 7-point Likert response format, ranging from 1 (strongly disagree) to 7 (strongly agree), with higher scores referring to greater coronavirus fear.

\section{Procedure}

An online survey was used to collect the data. The survey was developed with the Google form software and participants were recruited through emails or social media (e.g. WhatsApp, Facebook). A snowballing technique, whereby participants were encouraged to invite friends in similar circumstances to take part in the study, was used. Participants have been assured about anonymity and confidentiality of responses. Participants clicked the link in the advertisement and completed the survey following giving online informed consent. Institutional ethical approval was obtained prior to conducting the study.

\section{Data Analysis}

Using the whole sample without any missing values, we first examined preliminary analyses (e.g. mean, standard deviation), the internal reliability estimates, and the normality assumption. Subsequently, Pearson's correlation analysis was conducted next to examine the association between perceived risk, coronavirus fear, resilience, and psychological health problems. Kurtosis and skewness scores and their cut-off values were used to examine the assumption of normality (Kurtosis and skewness scores <|2|; D’Agostino et al. 1990). Mediation analyses were performed to test the mediating role of coronavirus fear and resilience in the association between perceived risk and psychological health problems using the PROCESS macro (Model 6) for SPSS version 3.4 (Hayes 2018). The bootstrapping method with 10,000 resamples to estimate the $95 \%$ confidence intervals (CI) was additionally conducted to investigate the significance of indirect effects (Hayes 2018). Significance level of $p<0.05$ was used for all analyses. 


\section{Results}

The findings of the preliminary analysis showed that skewness scores ranged from -.79 to 1.23, and kurtosis values were between -.62 and 1.32. These scores suggested that all variables had a normal distribution. Subsequently, Pearson's correlation analysis results revealed that perceived risk was significantly and positively correlated with coronavirus fear $(r=.54)$, depression $(r=.37)$, stress $(r=.31)$, and anxiety $(r=.33)$ as well as negatively associated with resilience $(r=-.19)$, as shown in Table 1. Resilience also had negative and significant correlation with coronavirus fear $(r=-.33)$, depression $(r=-.36)$, stress $(r=$ $-.43)$, and anxiety $(r=-.38)$. All correlation results were significant at the levels of $p<0.01$ (Tables 2 and 3 ).

Following examining the preliminary results and correlations, we tested the mediating role of the coronavirus fear and resilience in the association between perceived risk and psychological health problems including depression, stress, and anxiety. We first found that perceived risk significantly predicted coronavirus fear $(\beta=.54, p<.001)$ but was a non-significant predictor of resilience $(\beta=-.02, p=.84)$. Coronavirus fear fully mediated the effect of perceived risk on resilience $(\beta=-.32, p<.001)$. Perceived risk explained $29 \%$ of the variance in coronavirus fear, and perceived risk and coronavirus fear together accounted for $11 \%$ of the variance in resilience. Mediation analysis also showed that perceived risk was a significant predictor of depression $(\beta=.21, p<.001)$ and stress $(\beta=.15, p<.05)$ but was a nonsignificant predictor of anxiety $(\beta=-.09, p=.18)$. Coronavirus fear had a significant predictive effect on depression $(\beta=.22, p<.05)$, stress $(\beta=.18, p<.05)$, and anxiety $(\beta=.36$, $p<.001)$ and mediated the association between perceived risk and psychological health problems. Lastly, the results revealed that resilience mitigated the effect of coronavirus fear on depression $(\beta=-.25, p<.001)$, stress $(\beta=-.34, p<.001)$, and anxiety $(\beta=-.25, p<.001)$. All variables together explained $26 \%$ of the variance in depression, $26 \%$ of the variance in stress, and $26 \%$ of the variance in anxiety.

\section{Discussion}

The primary aim of this study was to investigate the potential mediating roles of coronavirus fear and resilience in the impact of perceived risk and mental health problems among healthcare professionals directly caring COVID-19 patients. Consistent with earlier research (Harper et al. 2020; Jackson 2011; Li et al. 2020), perceived risk had a direct effect on coronavirus fear and mental health problems, but not resilience. The results have supported the

Table 1 Descriptive statistics and correlation results

\begin{tabular}{|c|c|c|c|c|c|c|c|c|c|c|c|}
\hline Scales & $M$ & $S D$ & Skew. & Kurt. & $\alpha$ & 1. & 2. & 3. & 4. & 5. & 6. \\
\hline 1. Perceived risk & 7.62 & 1.62 & -.53 & -.25 & .78 & - & $.54^{* *}$ & $-.19^{* *}$ & $.37^{* *}$ & $.31^{* *}$ & $.33^{* *}$ \\
\hline 2. Coronavirus fear & 13.35 & 3.79 & -.79 & -.10 & .79 & & - & $-.33^{* *}$ & $.51^{* *}$ & $.37^{* *}$ & $.49^{* *}$ \\
\hline 3. Resilience & 19.95 & 4.69 & -.26 & .09 & .84 & & & - & $-.36^{* *}$ & $-.43^{* *}$ & $-.38^{* *}$ \\
\hline 4. Depression & 5.72 & 5.38 & 1.04 & .64 & .81 & & & & - & $.77^{* *}$ & $.63^{* *}$ \\
\hline 5. Stress & 7.24 & 5.68 & .53 & -.62 & .91 & & & & & - & $.59^{* *}$ \\
\hline 6. Anxiety & 4.59 & 3.98 & 1.23 & 1.32 & .91 & & & & & & - \\
\hline
\end{tabular}

** Correlation is significant at the .001 level (2-tailed) 
Table 2 Unstandardized coefficients for the mediation model

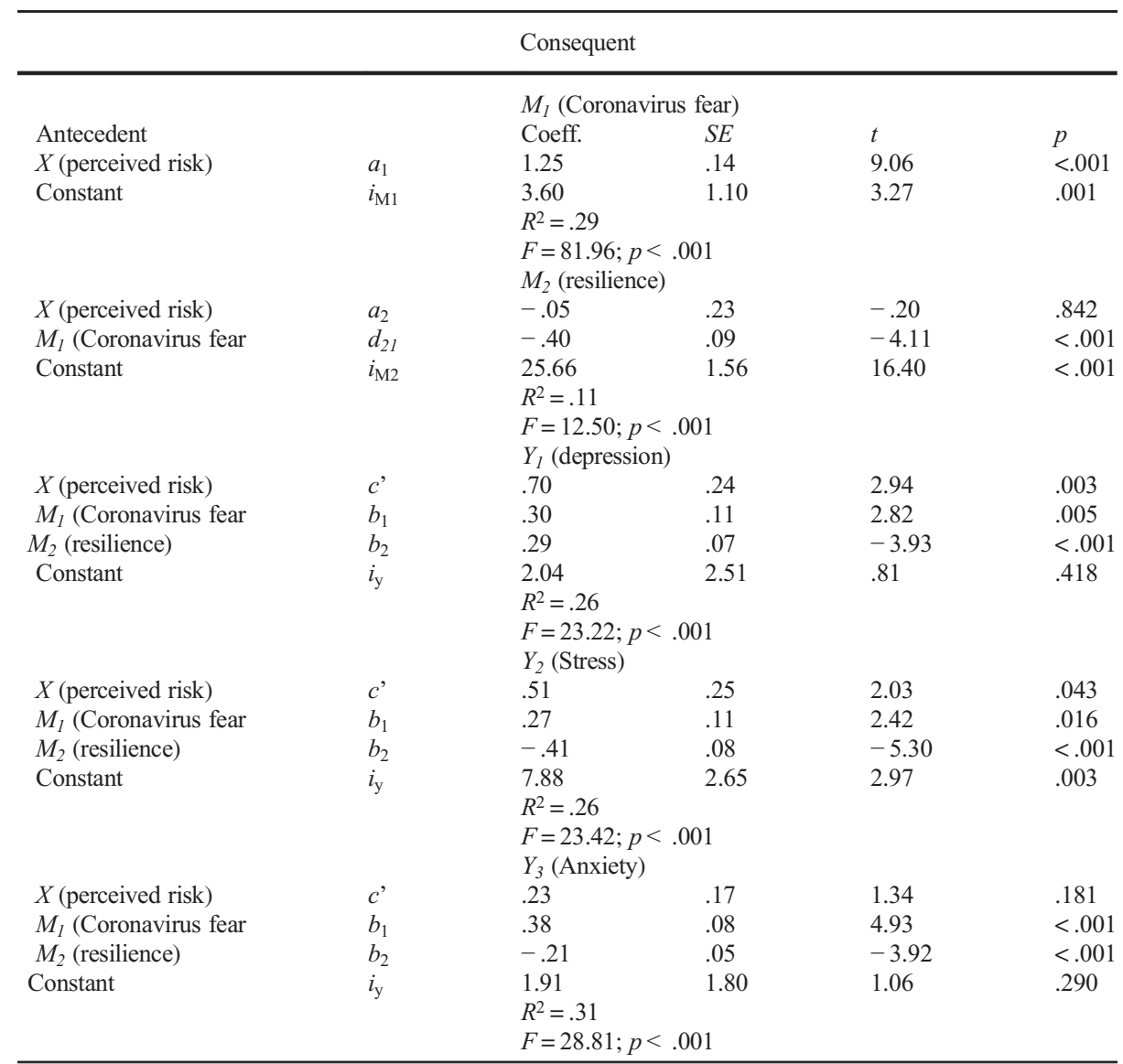

$S E$ standard error, Coeff unstandardized coefficient, $X$ independent variable, $M$ mediator variables, $Y$ outcomes or dependent variables

Table 3 Standardized indirect effects and 95\% bias-corrected confidence interval predicting psychological health scores

\begin{tabular}{lllll}
\hline Path & Effect & $S E$ & Boot LLCI & Boot ULCI \\
\hline Total indirect effect & .16 & .04 & .08 & .24 \\
Risk $\rightarrow$ fear->depression & .11 & .04 & .04 & .19 \\
Risk $\rightarrow$ resilience->depression & .01 & .02 & -.04 & .05 \\
Risk $\rightarrow$ fear->resilience-> depression & .01 & .02 & .02 & .08 \\
Total indirect effect & .16 & .04 & .08 & .24 \\
Risk $\rightarrow$ fear->stress & .10 & .04 & .03 & .18 \\
Risk $\rightarrow$ resilience->stress & .01 & .03 & -.05 & .06 \\
Risk $\rightarrow$ fear->resilience-> stress & .06 & .02 & .03 & .10 \\
Total indirect effect & .24 & .04 & .16 & .33 \\
Risk $\rightarrow$ fear $\rightarrow$ anxiety & .19 & .04 & .12 & .28 \\
Risk $\rightarrow$ resilience-> anxiety & .01 & .02 & -.03 & .05 \\
Risk $\rightarrow$ fear $\rightarrow$ resilience-> anxiety & .04 & .02 & .02 & .08 \\
\hline
\end{tabular}

Number of bootstrap samples for percentile bootstrap confidence intervals: 10,000 
study predictions that those who have high levels of perceived risk are more likely to experience fear, depression, anxiety, and stress. Although some early research has examined the association between risk and resilience (O'Gara et al. 2020), there is a scarcity of research investigating the mediating role of coronavirus fear between perceived risk and resilience. The current research found that coronavirus fear fully mediated the relationship between perceived risk and resilience, suggesting that the impact of perceived risk of COVID-19 on one's ability to bounce back from adversity is fully explained by the levels of coronavirus fear.

This study found that coronavirus fear had a direct effect on depression, stress, and anxiety. Prior research indicated that fear of illness or fear of the unknown has the potential to predict mental/physical health outcomes and negative societal behaviours such as psychological distress, mental health disorders, poor physical health, and health risk behaviours (Shigemura et al. 2020). Additionally, coronavirus fear significantly mediated the relationship between perceived risk and greater mental health problems. Those who have a higher perceived risk of COVID-19 may therefore experience more coronavirus fear that increases the risk of poor mental health. These results are in the line with those of previous research that coronavirus fear and perceived risk have significant impacts on mental health (Ahorsu et al. 2020; Li et al. 2020; Shigemura et al. 2020). Additionally, the present findings reported that resilience served as a significant mediator between coronavirus fear and mental health problems. These results are promising in terms of providing evidence of the protective role of resilience in mitigating the negative impact of coronavirus fear and psychological health of healthcare professionals. These results are in line with earlier studies on the association between resilience and mental health (Yildırım and Arslan 2020). The theoretical underpinning of this prediction is that excessive levels of fear related to COVID-19 may lead to poor mental health (Ahorsu et al. 2020; Garfin et al. 2020; Mertens et al. 2020; Shigemura et al. 2020; Sloan et al. 2020) and that the functional characteristics of resilient individuals may facilitate better mental health by buffering the impact of fear on mental health problems in the face of adversity (Bonanno 2004; Y1ldirım 2019).

This study contributes to the extant literature in the following ways. First, there is a scarcity of well-suited research on the associations between perceived risk, fear, resilience, and mental health problems among healthcare professionals. We found that perceived risk and fear can contribute to increased mental health problems among healthcare professionals, which may have temporal or long-term psychological consequences and tested mediating effects of resilience and fear between perceived risk and mental health problems. Although mediational studies do not test causality directly, they are very fruitful in terms of providing evidence that helps in changing therapeutic or intervention approaches (Windgassen et al. 2016). Investigating directionality and mechanism between the variables is a critical requirement for causality. Second, we expand on dearth of research investigating psychological factors associated with mental health of healthcare professionals during the pandemic. By including healthcare professionals, we extended previous research where the impact of COVID-19 on the general public has been predominantly studied (Yıldırım and Arslan 2020). Concerning practical implications, the current findings offer important evidence for the development of resilience-based interventions aiming to protect healthcare professionals' mental health, improve their quality of life, and provide a recommendation on public policies. Protecting the mental health of healthcare professionals from the risk of infections is crucial for them to effectually fight against COVID-19. Well-suited resilience-based intervention can be delivered online using social networking sites which conveniently provide the least physical contact with healthcare professionals to reduce the risk of coronavirus infection. Such interventions can aim 
to (a) support healthcare professionals to maintain their mental health so that they can continue working for providing primary care and health services in times of crisis without having psychological problems, and (b) determine healthcare professionals who may be vulnerable to stressors due to inability to cope with adversity during pandemic. Other than online interventions, psychiatric clinics can be effectively used to provide mental health services to healthcare workers who are actively involved in the fight against COVID-19 and develop symptoms of mental health problems such as anxiety and stress-related disorders (Chen et al. 2020).

We need to acknowledge some limitations of this research. First, although self-reports of one's experience are valid and feasible ways of measuring mental health problems and associated factors, exclusive reliance on self-reported measures may have the risk of source bias. Using alternative methods to supplement the present findings is suggested in future research. Second, given the cross-sectional nature of the study design, it was difficult to draw a conclusion regarding the cause and effect association between the study variables. Thus, longitudinal studies are necessary to confirm the causal order of the associations between our study variables. Such data are particularly fruitful with respect to offering the long-term mental health outcomes of the COVID-19 pandemic. Finally, the current findings should be replicated on other clinical and non-clinical samples to increase the generalisability of the findings.

Despite these limitations, the present findings might shed light on the possible mechanism underlying between perceived risk and mental health problems by considering the roles of resilience and fear in healthcare professionals. Findings may also provide directions to future research aiming to examine resilience and mental health within the context of crisis and implement resilience-based programmes.

Acknowledgements The authors would like to thank all participants involved in this study for their cooperation and support.

Data Availability Data are available from the corresponding author on a reasonable request.

\section{Compliance with Ethical Standards}

Conflict of Interest The authors declare that they have no conflict of interest.

Ethical Approval All procedures performed in studies involving human participants were in accordance with the ethical standards of the institutional and/or national research committee and with the 1964 Helsinki declaration and its later amendments or comparable ethical standards. The ethical approval for this study was obtained from Ağrı İbrahim Çeçen University Ethics Committee.

Informed Consent Consent was obtained from all participants included in the study.

\section{References}

Ahorsu, D. K., Lin, C. Y., Imani, V., Saffari, M., Griffiths, M. D., \& Pakpour, A. H. (2020). The fear of COVID19 scale: development and initial validation. International Journal of Mental Health and Addiction, 1-9. https://doi.org/10.1007/s11469-020-00270-8.

Arslan, G., Yıldırım, M., \& Wong, P. T. P. (2020). Meaningful living, resilience, affective balance, and psychological health problems during COVID-19. PsyArXiv. Preprint https://doi.org/10.31234/osf.io/wsr3e. 
Ashtari, S., Vahedian-Azimi, A., Moayed, M. S., Rahimibashar, F., Shojaei, S., \& Pourhoseingholi, M. A. (2020). Compare the severity of psychological distress among four groups of Iranian society in COVID-19 pandemic, 1-17. https://doi.org/10.21203/rs.3.rs-23828/v1.

Bonanno, G. A. (2004). Loss, trauma, and human resilience. American Psychologist, 59, 20-28.

Bults, M., Beaujean, D. J., de Zwart, O., Kok, G., van Empelen, P., van Steenbergen, J. E., Richardus, J. H., \& Voeten, H. A. (2011). Perceived risk, anxiety, and behavioural responses of the general public during the early phase of the influenza A (H1N1) pandemic in the Netherlands: results of three consecutive online surveys. BMC Public Health, 11(1), 2.

Cai, H., Tu, B., Ma, J., Chen, L., Fu, L., Jiang, Y., \& Zhuang, Q. (2020). Psychological impact and coping strategies of frontline medical staff in Hunan between January and March 2020 during the outbreak of coronavirus disease 2019 (COVID-19) in Hubei, China. Medical science monitor: International Medical Journal of Experimental and Clinical Research, 26, e924171-1-e924171-16.

Center for Systems Science and Engineering. (2020). Coronavirus COVID-19 global cases at Johns Hopkins University. Retrieved from https://coronavirus.jhu.edu/map.html.

Chen, Q., Liang, M., Li, Y., Guo, J., Fei, D., Wang, L., ... \& Wang, J. (2020). Mental health care for medical staff in China during the COVID-19 outbreak. The Lancet Psychiatry, 7(4), e15-e16.

Cheng, S. K. W., Chong, G. H. C., Chang, S. S. Y., Wong, C. W., Wong, C. S. Y., Wong, M. T. P., \& Wong, K. C. (2006). Adjustment to severe acute respiratory syndrome (SARS): Roles of appraisal and post-traumatic growth. Psychology \& Health, 21(3), 301-317. https://doi.org/10.1080/14768320500286450.

D’Agostino, R. B., Belanger, A., \& D’Agostino, R. B. (1990). A suggestion for using powerful and informative tests of normality. The American Statistician, 44(4), 316. https://doi.org/10.2307/2684359.

Doğan, T. (2015). Adaptation of the Brief Resilience Scale into Turkish: a validity and reliability study. The Journal of Happiness \& Well-Being, 3(1), 93-102.

Fertleman, C., \& Carroll, W. (2013). Protecting students and promoting resilience. BMJ, 347, f5266.

Garfin, D. R., Silver, R. C., \& Holman, E. A. (2020). The novel coronavirus (COVID-2019) outbreak: amplification of public health consequences by media exposure. Health Psychology, 39, 355-357. https://doi.org/10.1037/hea0000875.

Harper, C. A., Satchell, L. P., Fido, D., \& Latzman, R. D. (2020). Functional fear predicts public health compliance in the COVID-19 pandemic. International Journal of Mental Health and Addiction, 1-14. https://doi.org/10.1007/s11469-020-00281-5.

Hayes, A. F. (2018). Introduction to mediation, moderation, and conditional process analysis: a regressionbased approach. New York: Guilford Press.

Huang, Y., \& Zhao, N. (2020). Mental health burden for the public affected by the COVID-19 outbreak in China: who will be the high-risk group? Psychology, Health \& Medicine, 1-12. https://doi.org/10.1080 /13548506.2020.1754438.

Jackson, J. (2011). Revisiting risk sensitivity in the fear of crime. Journal of Research in Crime and Delinquency, 48(4), 513-537.

Janz, N. K., \& Becker, M. H. (1984). The health belief model: a decade later. Health Education Quarterly, 11(1), $1-47$.

Khosravi, M. (2020). Perceived risk of COVID-19 pandemic: the role of public worry and trust. Electronic Journal of General Medicine, 17(4), em203.

Kwok, K. O., Li, K. K., Chan, H. H., Yi, Y. Y., Tang, A., Wei, W. I., \& Wong, S. Y. (2020). Community responses during early phase of covid-19 epidemic, Hong Kong. Emerging Infectious Diseases, 26(7), 1-13.

Lancee, W. J., Maunder, R. G., \& Goldbloom, D. S. (2008). Prevalence of psychiatric disorders among Toronto hospital workers one to two years after the SARS outbreak. Psychiatric Services, 59(1), 91-95.

Li, J. B., Yang, A., Dou, K., \& Cheung, R. Y. (2020). Self-control moderates the association between perceived severity of the coronavirus disease 2019 (COVID-19) and mental health problems among the Chinese public. PsyArXiv Preprints. https://doi.org/10.31234/osf.io/2xadq.

Lovibond, P. F., \& Lovibond, S. H. (1995). The structure of negative emotional states: comparison of the Depression Anxiety Stress Scales (DASS) with the Beck Depression and Anxiety inventoRies. Behaviour Research and Therapy, 33(3), 335-343.

Masten, A. S. (2001). Ordinary magic. Resilience processes in development. The American Psychologist, 56(3), 227-238. https://doi.org/10.1037/0003-066x.56.3.227.

Mertens, G., Gerritsen, L., Salemink, E., \& Engelhard, I. (2020). Fear of the coronavirus (COVID-19): predictors in an online study conducted in March 2020. https://doi.org/10.31234/osf.io/2p57j.

O'Gara, J. L., Calzada, E. J., \& Kim, S. Y. (2020). The father's role in risk and resilience among MexicanAmerican adolescents. American Journal of Orthopsychiatry, 90(1), 70-77.

Ong, A. D., Bergeman, C. S., Bisconti, T. L., \& Wallace, K. A. (2006). Psychological resilience, positive emotions, and successful adaptation to stress in later life. Journal of Personality and Social Psychology, 91(4), 730-749. 
Peeri, N. C., Shrestha, N., Rahman, M. S., Zaki, R., Tan, Z., Bibi, S., ... \& Haque, U. (2020). The SARS, MERS and novel coronavirus (COVID-19) epidemics, the newest and biggest global health threats: what lessons have we learned? International Journal of Epidemiology, dyaa033, https://doi.org/10.1093/ije/dyaa033

Perin, C., Beghi, M., Cerri, C. G., Peroni, F., Viganò, B., \& Cornaggia, C. M. (2015). Experience of group conversations in rehabilitative medicine: methodological approach and pilot study. Journal of Medicine and the Person, 13, 96-104. https://doi.org/10.1007/s12682-015-0208-7.

Robertson, H. D., Elliott, A. M., Burton, C., Iversen, L., Murchie, P., Porteous, T., \& Matheson, C. (2016). Resilience of primary healthcare professionals: a systematic review. British Journal of General Practice, 66(647), e423-e433.

Sariçam, H. (2018). The psychometric properties of Turkish version of Depression Anxiety Stress Scale-21 (DASS-21) in health control and clinical samples. Journal of Cognitive Behavioral Psychotherapy and Research, 7, 19-30.

Shigemura, J., Ursano, R. J., Morganstein, J. C., Kurosawa, M., \& Benedek, D. M. (2020). Public responses to the novel 2019 coronavirus $(2019-\mathrm{nCoV})$ in Japan: mental health consequences and target populations. Psychiatry and Clinical Neurosciences, 74(4), 281-282.

Sloan, M. M., Haner, M., Graham, A., Cullen, F. T., Pickett, J., \& Jonson, C. L. (2020). Pandemic emotions: the extent, correlates, and mental health consequences of personal and altruistic fear of COVID-19[Preprint]. SocArXiv. https://doi.org/10.31235/osf.io/txqb6.

Smith, B. W., Dalen, J., Wiggins, K., Tooley, E., Christopher, P., \& Bernard, J. (2008). The brief resilience scale: assessing the ability to bounce back. International Journal of Behavioral Medicine, 15(3), 194-200.

Stevenson, A. D., Phillips, C. B., \& Anderson, K. J. (2011). Resilience among doctors who work in challenging areas: a qualitative study. British Journal of General Practice, 61(588), e404-e410.

Tan, B. Y., Chew, N. W., Lee, G. K., Jing, M., Goh, Y., Yeo, L. L., ... \& Shanmugam, G. N. (2020). Psychological impact of the COVID-19 pandemic on health care workers in Singapore. Annals of Internal Medicine. M20-1083. https://doi.org/10.7326/M20-1083.

Tecson, K. M., Wilkinson, L. R., Smith, B., \& Ko, J. M. (2019). Association between psychological resilience and subjective well-being in older adults living with chronic illness. In Baylor University Medical Center Proceedings (Vol. 32, no. 4, pp. 520-524). Taylor \& Francis.

Warr, M. (2000). Fear of crime in the United States: Avenues for research and policy. Criminal Justice, 4(4), $451-489$.

Weinstein, N. D. (1982). Unrealistic optimism about susceptibility to health problems. Journal of Behavioral Medicine, 5(4), 441-460.

Windgassen, S., Goldsmith, K., Moss-Morris, R., \& Chalder, T. (2016). Establishing how psychological therapies work: the importance of mediation analysis. Journal of Mental Health, 25(2), 93-99.

Wise, T., Zbozinek, T., Michelini, G., Hagan, C. C., \& Mobbs, D. (2020). Changes in risk perception and protective behavior during the first week of the COVID-19 pandemic in the United States. PsyArXiv Preprints. https://doi.org/10.31234/osf.io/dz428.

Yang, J. Z., \& Chu, H. R. (2018). Who is afraid of the Ebola outbreak? The influence of discrete emotions on risk perception. Journal of Risk Research, 21(7), 834-853. https://doi.org/10.1080/13669877.2016.1247378.

Yildırım, M. (2019). Mediating role of resilience in the relationships between fear of happiness and affect balance, satisfaction with life, and flourishing. Europe's Journal of Psychology, 15(2), 183-198.

Yıldırım, M., \& Arslan, G. (2020). Exploring the associations between resilience, dispositional Hope, preventive behaviours, subjective well-being, and psychological health among adults during early stage of COVID-19. PsyArXiv. Preprint https://doi.org/10.31234/osf.io/vpu5q10.31234/osf.io/vpu5q.

Publisher's Note Springer Nature remains neutral with regard to jurisdictional claims in published maps and institutional affiliations. 